hep-th/9510229

MRI-PHY $/ 23 / 95$

\title{
A NOTE ON MARGINALLY STABLE BOUND STATES IN TYPE II STRING THEORY
}

\author{
Ashoke Sen円苗 \\ Mehta Research Institute of Mathematics \\ and Mathematical Physics \\ 10 Kasturba Gandhi Marg, Allahabad 211002, INDIA
}

\begin{abstract}
Spectrum of elementary string states in type II string theory contains ultra-short multiplets that are marginally stable. $U$-duality transformation converts these states into bound states at threshold of $p$-branes carrying Ramond-Ramond charges, and wrapped around $p$-cycles of a torus. We propose a test for the existence of these marginally stable bound states. Using the recent results of Polchinski and of Witten, we argue that the spectrum of bound states of $p$-branes is in agreement with the prediction of $U$-duality.
\end{abstract}

\footnotetext{
${ }^{1}$ On leave of absence from Tata Institute of Fundamental Research, Homi Bhabha Road, Bombay 400005, INDIA

${ }^{2}$ E-mail: sen@mri.ernet.in, sen@theory.tifr.res.in
} 
The spectrum of type IIA or IIB string theory compactified on a torus contains single particle states in the ultra-short (256 dimensional) multiplet. These states are characterized by the following two properties: (1) the left and the right moving charges carried by these states are equal in magnitude, and (2) both, the left and the right moving oscillators are in their ground state. All such states come with unit multiplicity, i.e. for a given set of charge quantum numbers satisfying the property (1) above, there is only one ultra-short multiplet. Some of these states are marginally stable, i.e. it is energetically possible for these states to decay into two or more single particle states at rest [1]. This happens if the electric charge vector, represented by an element of the charge lattice, is an integral multiple of another vector in the lattice. Examples of such states are 1) the ground state of a string wrapped $m$ times around one of the cycles on the torus, 2 ) the Kaluza-Klein modes of the massless states of the ten dimensional theory carrying $m$ units of momentum along one of the compact directions, etc.

Consider now the type II theory compactified on a $p$ dimensional torus $T^{p}$. We shall choose $p$ to be odd for the type IIB theory and even for the type IIA theory, for reasons that will become clear soon. In this case we can consider a supersymmetric soliton solution of the theory that corresponds to a $p$-brane carrying Ramond-Ramond (RR) charge 2] (which exists in the IIA theory for even $p$ and in the IIB theory for odd $p$ ) wrapped $m$-times around the $p$-cycle of the torus. For definiteness we shall choose $x^{\mu}$ for $(10-p) \leq \mu \leq 9$ to be the compact directions. If we denote by $\mathcal{A}_{\mu_{1} \cdots \mu_{p+1}}^{(p+1)}$ the $(p+1)$-form gauge field arising in the RR sector, then the $p$-brane described above is charged under the $(10-p)$ dimensional gauge field $\mathcal{A}_{(10-p) \cdots 89 \bar{\mu}}^{(p+1)}$ where $x^{\bar{\mu}}$ denotes one of the non-compact directions $(0 \leq \bar{\mu} \leq(9-p))$. In fact, this solution carries $m$ units of $\mathcal{A}_{(10-p) \cdots 89 \bar{\mu}}^{(p+1)}$ charge. We shall denote by $Q^{(p+1)}$ the charge associated with the field $\mathcal{A}_{(10-p) \cdots 89 \bar{\mu}}^{(p+1)}$, normalized so that $Q^{(p+1)}$ is always an integer. The question that we shall be interested in is: 'Are there any ultra-short multiplets in the sector $Q^{(p+1)}=m$; and if so, how many of them are present for a given value of $m$ ?'

A prediction for the degeneracy of these states is given by the general $U$-duality conjecture of Hull and Townsend[3]. It can be easily seen that by combining the $T$ duality transformations (which sometimes convert a type IIB theory to a type IIA theory and vice versa) with the SL(2,Z) symmetry of the ten dimensional type IIB theory, we can transform the field $\mathcal{A}_{(10-p) \cdots 89 \bar{\mu}}^{(p+1)}$ to $B_{9 \bar{\mu}}$, where $B_{\mu \nu}$ denotes the rank two anti-symmetric tensor field arising in the NS-NS sector. Since $B_{9 \bar{\mu}}$ couples to the winding number of the fundamental string in the 9 th direction, we see that the duality transformation discussed above transforms a state of $Q^{(p+1)}=m$ to a state of the fundamental string winding $m$ times around the 9 th direction. Since we already know that a fundamental string winding $m$ times around $x^{9}$ has in its spectrum an ultra-short multiplet, there must also be an ultra-short multiplet with $Q^{(p+1)}=m$ if $U$-duality is a valid symmetry of string theory. In future we shall refer to ultra-short multiplets as BPS states.

The BPS states with $Q^{(p+1)}=m$ can be thought of as representing bound states of $m$ elementary $p$-branes, each wrapped only once around the $p$-cycle. Studying the existence of these bound states is made difficult by the fact that they have zero binding energy. In other words there is no energy barrier against pulling the $m p$-branes away

\footnotetext{
${ }^{3}$ Consider, for example the duality transformation that inverts the radii of the $(p-1)$ circles labelled by coordinates $x^{10-p}, \ldots x^{8}$. This would convert $\mathcal{A}_{(10-p) \cdots 89 \bar{\mu}}^{(p+1)}$ to $B_{9 \bar{\mu}}^{\prime}$ where $B_{\mu \nu}^{\prime} \equiv \mathcal{A}_{\mu \nu}^{(2)}$ is the rank two anti-symmetric tensor field in the IIB theory arising in the RR sector. With the help of the conjectured $\mathrm{SL}(2, \mathrm{Z})$ symmetry transformation in the ten dimensional type IIB string theory, we can convert $B_{9 \bar{\mu}}^{\prime}$ to $B_{9 \bar{\mu}}$.
} 
from each other. A related problem is that since these states have exactly the same energy as $m$ BPS states - each with $Q^{(p+1)}=1$ - at rest, it is difficult to separate this single particle state from the continuum. f For these reasons we shall adopt an indirect approach for studying the existence of these bound states.

Suppose an ultra-short multiplet carrying $Q^{(p+1)}=m$ does exist in the $(10-p)$ dimensional theory obtained by compactifying the type II theory on $T^{p}$. Let us now compactify one more direction (which we shall take to be the direction $x^{1}$ for definiteness) on a circle of radius $R$. Then the original ultra-short multiplet will give rise to an infinite number of ultra-short multiplets in this $(9-p)$ dimensional theory, - one for each value of the internal momentum along the $x^{1}$ direction. Let us denote by $P^{1}$ the internal momentum along $x^{1}$, normalized so that it is always an integer, and focus on a state with $P^{1}=n$, where $n$ is any arbitrary integer. If we choose $n$ in such a way that $n$ and $m$ are relatively prime, then the ultra-short multiplet in the $\left(Q^{(p+1)}=m, P^{1}=n\right)$ sector becomes absolutely stable, even though the original ultra-short multiplet in the $(10-p)$ dimensional theory was only marginally stable. Thus one of the consequences of the existence of marginally stable ultra-short multiplets in $(10-p)$ dimensional theory, carrying $Q^{(p+1)}$ charge $m$, is that in the $(9-p)$ dimensional theory there must exist absolutely stable ultra-short multiplets with $Q^{(p+1)}=m$, and $P^{1}=n$, for every integer $n$ for which $(m, n)$ are relatively prime. The existence of the latter states will be much easier to verify. In fact, these states can be mapped to the winding modes of the $(m, n)$ string discussed in refs. [5, 6] via $T$-duality transformation. Consider for example the $T$-duality transformation that inverts the radii of all the $p+1$ circles labelled by $x^{1}, x^{(10-p)}, \ldots x^{9}$. This transforms $G_{1 \mu}$ to $B_{1 \mu}$ and $\mathcal{A}_{(10-p) \ldots 9 \bar{\mu}}^{(p+1)}$ to $B_{1 \bar{\mu}}^{\prime} \equiv \mathcal{A}_{1 \bar{\mu}}^{(2)}$. Thus the $\left(Q^{(p+1)}=m, P^{1}=n\right)$ state is mapped to an $(m, n)$ string wrapped around the $x^{1}$ direction. The existence of $(m, n)$ strings for relatively prime $m, n$ has already been shown in ref. [6]; this then guarantees the existence of $\left(Q^{(p+1)}=m, P^{1}=n\right)$ states. We shall now see explicitly how it works.

It has recently been shown by Polchinski[7] that there is an exact conformal field theory description of a $p$-brane carrying $\mathrm{RR}$ charges. This is given by a Dirichlet brane, a $p$ dimensional hypersurface such that open strings satisfy Dirichlet boundary conditions for coordinates transverse to the hypersurface, and Neumann boundary condition for coordinates tangential to the hypersurface. Let us apply this to the case of a static $p$ brane wrapped once around a $p$-torus labelled by the coordinates $x^{\mu}$ for $(10-p) \leq \mu \leq 9$, situated at the point $x^{\mu}=a^{\mu}$ for $1 \leq \mu \leq(9-p)$. The open strings then satisfy the boundary condition:

$$
\begin{aligned}
\partial_{\sigma} X^{\mu} & =0 \quad \text { for }(10-p) \leq \mu \leq 9, \\
X^{\mu} & =a^{\mu} \quad \text { for } 1 \leq \mu \leq(9-p), \\
\partial_{\sigma} X^{0} & =0,
\end{aligned}
$$

where $\sigma$ denotes the coordinate along the length of the string. If we further compactify the coordinate $x^{1}$ along a circle of radius $R$, then the open string boundary condition on $X^{1}$ will be modified to,

$$
X^{1}=a^{1} \quad \bmod \quad 2 \pi R .
$$

The generalization to the case of multiple $p$-branes is straightforward. If $\left\{a_{i}^{\bar{\mu}}\right\}(1 \leq i \leq m$, $1 \leq \bar{\mu} \leq(9-p))$ denote the coordinates of the $m p$-branes, then we have $m^{2}$ different sectors of open string states, with each end being allowed to lie on any of the $m p$-branes.

\footnotetext{
${ }^{4}$ However, in $N=2$ supersymmetric gauge theories this difficulty has been circumvented, and one does find such marginally stable bound states, as predicted by duality 4 .
} 
We want to study if the quantization of the classical solution representing this multi$p$-brane solution gives rise to a BPS state carrying $n$ units of momentum in the $x^{1}$ direction. For this it will be convenient to map this problem to another problem by a $T$-duality transformation that inverts the radius $R$ in the $x^{1}$ direction. This has several effects: 1 ) it converts a type IIB theory to a type IIA theory and vice versa, 2) it converts the Dirichlet boundary condition on the coordinate $X^{1}$ to Neumann boundary condition [8], 3 ) it converts the quantum number representing momentum along $x^{1}$ to winding number, and 4) it converts $\mathcal{A}_{(10-p) \ldots 9 \bar{\mu}}^{(p+1)}$ to $\mathcal{A}_{1(10-p) \ldots 9 \bar{\mu}}^{(p+2)}$, and hence $Q^{(p+1)}$ to $Q^{(p+2)}$. Thus if we denote by $y^{\mu}$ the space-time coordinates in the transformed theory, then the new conformal field theory is described by the following open string boundary conditions:

$$
\begin{aligned}
& \partial_{\sigma} Y^{\mu}=0 \quad \text { for }(10-p) \leq \mu \leq 9 \text {, } \\
& \partial_{\sigma} Y^{0}=0 \\
& \partial_{\sigma} Y^{1}=0 \\
& Y^{\mu}=a_{i}^{\mu} \quad \text { for } 2 \leq \mu \leq(9-p),
\end{aligned}
$$

where $1 \leq i \leq m . y^{1}$ is a compact coordinate with radius $R^{-1}$, whereas $y^{\mu}$ for $(10-p) \leq$ $\mu \leq 9$ are compact coordinates labelling the original torus $T^{p}$. The rest of the $y^{\mu}$ are non-compact.f This conformal field theory represents $m(p+1)$-branes, each wrapped around the $p+1$ torus $T^{p+1}$ labelled by the coordinates $y^{1}$, and $y^{\mu}$ for $(10-p) \leq \mu \leq 9$. We want to look for BPS states of this system which carry $n$ units of winding number along the $y^{1}$ direction. For this we need to know the dynamics of collective coordinates of this system. This has been given recently in a beautiful paper by Witten [6] where he shows that the low energy dynamics of this system is described by a supersymmetric $U(m)$ gauge theory in $(p+2)$ dimensions, obtained by dimensional reduction of the $N=1$ supersymmetric $U(m)$ gauge theory in 10 dimensions. The base manifold here is $R \times T^{p+1}$ where $R$ is labelled by the time coordinate $y^{0}$, and $T^{p+1}$ by the coordinates $y^{1}$ and $y^{\mu}$ $((10-p) \leq \mu \leq 9)$. Furthermore a state carrying $n$ units of winding along the $y^{1}$ direction corresponds to a state in this $U(m)$ gauge theory characterized by $n$ units of $U(1)$ electric flux along the $y^{1}$ direction. (This in turn implies that there is also $S U(m)$ electric flux along the $y^{1}$ direction in the representation corresponding to the anti-symmetric product of $n$ fundamental representations.)

Since we are interested in only the BPS states of the system, and in only those states that do not carry any quantum numbers representing momentum and winding in the directions $y^{\mu}$ for $(10-p) \leq \mu \leq 9$, we can ignore fluctuations in the fields that depend non-trivially on any of these directions. In other words, for our purpose we can consider dimensional reduction of the $(p+2)$ dimensional theory to a two dimensional theory labelled by the coordinates $y^{0}$ and $y^{1}$. This corresponds to $N=8$ supersymmetric $U(m)$ gauge theory in $1+1$ dimensions, and is precisely the theory that has been analyzed in ref. [6]. 9

\footnotetext{
${ }^{5}$ Note that the parameters $\left\{a_{i}^{1}\right\}$ have disappeared from eq.(3), but they will reappear as components of the gauge field along the $y^{1}$ direction when we take into account the collective coordinates of this solution.

${ }^{6}$ Although it seems that the theory we have obtained this way is independent of $p$, there is, in fact, a subtle dependence on $p$. The theory has eight matrix valued scalar fields in the adjoint representation of $U(m) . p$ of these fields come from the internal components of the gauge fields in the $p+2$ dimensional theory. It was shown in ref. [9] that some of the directions in the field space (corresponding to the internal components of the gauge fields) need to be periodically identified. This introduces a dependence of the theory on $p$. Since we are interested only in the question of existence of supersymmetric ground states in the theory, and since the supersymmetric ground states of the theory found in ref. [6] are well localized in the field space, we expect that the extra periodic identification in the field space will not destroy the supersymmetric ground states found in [6].
} 
Following [6] we see that the problem of proving the existence of the required bound state reduces to the problem of proving the existence of a supersymmetric ground state in the $\mathrm{N}=8$ supersymmetric $S U(m)$ gauge theory in two dimensions, in the sector that carries an electric flux along the space direction $y^{1}$ (which in this case is compact) in the representation corresponding to anti-symmetric product of $n$ fundamental representations of $S U(m)$. It has already been argued in ref. [6] that such states do exist for every pair of integers $(m, n)$ which are relatively prime. This in turn establishes the existence of the $p$-brane bound states in the $(9-p)$ dimensional string theory, carrying $m$ units of $Q^{(p+1)}$ charge, and $n$ units of momentum along the $x^{1}$ direction, for every pair of relatively prime integers $(m, n)$.

As has been argued before, this is a necessary condition for the existence of a marginally stable $p$-brane bound state in $(10-p)$ dimensions, carrying $m$ units of $Q^{(p+1)}$ charge. One might ask if it is possible to see these bound states by working directly in the $(10-p)$ dimensional theory. In this case, there is no need to compactify the $x^{1}$ coordinate, and we can work directly with the Dirichlet $p$-brane described in eq.(1). The low energy dynamics of $m$ such $p$-branes is given, according to ref. [6], by a supersymmetric $U(m)$ gauge theory in $p+1$ dimensions, obtained by dimensional reduction of the $N=1$ supersymmetric $U(m)$ gauge theory in $9+1$ dimensions. The base space of this $p+1$ dimensional theory is $T^{p} \times R$, labelled by the space coordinates $x^{\mu}$ for $(10-p) \leq \mu \leq 9$, and the time coordinate $x^{0}$. As in ref. [6], the $U(1)$ part of the $U(m)$ theory is responsible for describing the overall center of mass motion of the state, as well as for producing the 256 fold degeneracy that is appropriate for an ultra-short multiplet. Thus the nontrivial information comes from the $S U(m)$ part of the theory, and the problem of counting the number of ultra-short multiplets reduces to the problem of counting the number of supersymmetric ground states of the supersymmetric $S U(m)$ gauge theory on $T^{p} \times R$.

In this case since we are interested in states which carry only the charge $Q^{(p+1)}$, and no other winding or momenta in any of the internal directions, the sector of the supersymmetric gauge theory that we need to analyze does not carry any background $S U(m)$ electric field. As a result, for studying supersymmetric ground states of the theory we can ignore fluctuations in the fields that depend on any of the $p$ compact coordinates and consider dimensional reduction of the theory to $(0+1)$ dimensions. Thus the problem under consideration reduces to a supersymmetric quantum mechanics problem obtained by dimensionally reducing the $N=1$ supersymmetric Yang-Mills theory from $(9+1)$ dimensions to $(0+1)$ dimension. The number of normalizable supersymmetric ground states of this system will be in one to one correspondence with the number of ultrashort multiplets in the type II theory on $T^{p}$ carrying $Q^{(p+1)}$ charge $m$. Since $U$-duality predicts that the latter number is one, we arrive at the following conclusion: The quantum mechanical system, obtained by dimensional reduction of the $N=1$ super Yang-Mills theory with gauge group $S U(m)$ from $(9+1)$ dimension to $(0+1)$ dimension, should have a unique normalizable supersymmetric ground state.

Explicit verification of this prediction is made complicated by the fact that there is no energy barrier that prevents a zero energy state from spreading out to infinity. As a result, even if a normalizable super-symmetric ground state exists, it will only decay according to a power law, and not exponentially for large separation. Note again that a subtle dependence on $p$ arises from the fact that for non-zero $p$ some of the directions in the space of scalar fields need to be periodically identified.

For $p=0$, the marginally stable states that we are looking for correspond to point like states in 10 dimensional type IIA string theory, carrying $m$ units of $A_{\mu} \equiv \mathcal{A}_{\mu}^{(1)}$ charge [6]. The existence of these states is essential for establishing that the strong cou- 
pling limit of the type IIA theory in ten dimensions is described by the 11-dimensional supergravity theory compactified on a circle of large radius 10.

It should be possible to carry out a similar analysis for more general compactification of the type II theory, on $K 3$ surfaces or on Calabi-Yau 3-folds. The expected degeneracy of ultra-short multiplets for compactification of the type IIA / IIB theory on $K 3$ surfaces may be worked out in some cases using the string-string duality conjecture[3] / the SL(2,Z) symmetry of the ten dimensional type IIB theory. For example, if we consider a two-brane wrapped $m$ times around a two cycle of $K 3$ that does not self intersect, then the BPS states in this sector will be mapped to the BPS states in the heterotic string theory with

$\vec{Q}_{L}^{2}=\vec{Q}_{R}^{2}\left(N_{L}=1\right)$. In this case we expect 24 (16-dimensional) BPS super-multiplets for every integer $m$. For two branes wrapped around two cycles which self-intersect, the answer will be more complicated. For compactification on Calabi-Yau 3-folds, one can get information about the expected degeneracy of the short multiplets by either requiring consistent resolution of the conifold singularity 11] or by using string-string duality conjecture 12]. It will be interesting to see if one can explicitly verify some of these predictions.

I wish to thank J. Schwarz for useful correspondence.

\section{References}

[1] A. Dabholkar and J. Harvey, Phys. Rev. Lett. 63 (1989) 478.

[2] G. Horowitz and A. Strominger, Nucl. Phys. B360 (1991) 197.

[3] C. Hull and P. Townsend, Nucl. Phys. B438 (1995) 109 (hep-th/9410167).

[4] S. Sethi, M. Stern and E. Zaslow, preprint hep-th/9508117;

J. Harvey and J. Gauntlett, preprint hep-th/9508156.

[5] J. Schwarz, preprints hep-th/9508143, hep-th/9509148, hep-th/9510086.

[6] E. Witten, preprint hep-th/9510135.

[7] J. Polchinski, preprint hep-th/9510017.

[8] J. Dai, R. Leigh and J. Polchinski, Mod. Phys. Lett. A4 (1989) 2073;

R. Leigh, Mod. Phys. Lett. A4 (1989) 2767;

P. Horava, Nucl. Phys. B327 (1989) 461; Phys. Lett. B321 (1989) 251;

M. Green, Phys. Lett. B329 (1994) 435;

J. Polchinski, Phys. Rev. D50 (1994) 6041 (hep-th/9407031).

[9] E. Witten, Nucl. Phys. B202 (1982) 253.

[10] E. Witten, Nucl. Phys. B443 (1995) 85 (hep-th/9503124);

P. Townsend, Phys. Lett. B350 (1995) 184 (hep-th/9501068).

[11] A. Strominger, Nucl. Phys. B451 (1995) 96 (hep-th/9504090).

[12] S. Kachru and C. Vafa, preprint hep-th/9505105. 